\title{
Slip-line field modeling of orthogonal machining pressure sensitive materials
}

\author{
G. G. Ye • S. F. Xue $・$ X. H. Tong $\cdot$ L. H. Dai
}

Received: 9 July 2010 / Accepted: 13 June 2011 /Published online: 28 June 2011

(C) Springer-Verlag London Limited 2011

\begin{abstract}
The slip-line field methods are widely used in solving cutting problem; however, most of which were focused on the pressure-independent materials. In this work, a new slip-line field model for orthogonal cutting of pressure sensitive materials is developed. Analytical characterization for orthogonal cutting process is obtained, which can give the explicit expressions for the shear angle, cutting force, and chip thickness in terms of the tool geometry, the friction coefficients on the tool flat, and the internal friction angle of the materials. To investigate the effect of the material and cutting parameters on cutting process, the finite element simulation is performed as well. The comparisons between the shear angle and cutting force predicted by the theoretical model with those obtained from finite element model simulation are made. The good agreement of the predicted results with the numerical results clearly reveals that the proposed slip-line field model can satisfactorily characterize the orthogonal cutting behavior of the pressure sensitive materials. Further analysis has demonstrated that the pressure sensitivity of materials has a significant influence on cutting process.
\end{abstract}

Keywords Orthogonal cutting · Slip-line field · Pressure sensitivity. Shear plane

G. G. Ye $\cdot$ S. F. Xue $\cdot$ X. H. Tong

Department of Engineering Mechanics,

Petroleum University of China,

Shan Dong 257061, China

L. H. Dai $(\bowtie)$

State Key Laboratory of Nonlinear Mechanics,

Institute of Mechanics, Chinese Academy of Sciences,

Beijing 100190, China

e-mail: lhdai@lnm.imech.ac.cn

\section{Introduction}

Metal cutting is the common manufacturing process used to remove unwanted materials from a workpiece and obtain designed geometrical dimensions and surface finish. This progress can be characterized by large strains, high strain rates, high stresses and temperatures, and unusual frictional conditions. Because of its great economic and technical importance, quantitatively estimating the technological performance of machining operations such as shear angle, forces, stresses, and the energy is important for the optimization of cutting conditions for the efficient and effective use of machining operations. Therefore, considerable efforts have been carried out in this field during past decades and several classical mechanical models have been developed, see, for example, the fairly comprehensive review books of Atkins [1], Shaw [2], and Oxley [3].

Piispanen [4] introduced the well-known "deck of cards" model in which the process was suggested to be one of concentrated shear on a plane extending upward from the tool point to the free surface, and this ideas also appeared in the Merchant's $[5,6]$ analysis that culminated in the development of a condensed force diagram which is popularly known as "Merchant Circle". For all this models, the solutions are obtained by assuming that the shear angle would be such as to minimize the work done in machining. Recently, Atkins [7, 8] demonstrated that the work associated with material separation cannot be neglected and proposed a new orthogonal cutting model by considering fracture toughness as the specific energy of surface formation in addition to the energy expended in shear and friction. By using this model, the material-dependent shear angle is obtained, and the "size effect" phenomena in cutting can be effectively characterized [9]. 
It is well known that the problems during machining not only come from the mechanical but also the kinematic aspects. It is noted that the slip-line field model can simultaneously satisfy both the stress equilibrium and velocity requirements of the flow of the chip material. Moreover, compared to other methods such as the minimum energy principle, the slip-line theory can show much more clearly the flow pattern of chip material over the whole range of the shear deformation zone (SDZ). The extremely complicated flow of the chip material taking place over the SDZ is one of the most basic and important characteristics of machining processes. It can be said that the characteristics of machining processes can be well understood so long as the rules of the flow of the chip material are known. Therefore, the modeling methodology based on slip-line theory has been widely employed and several slip-line models have been developed in the past decades. Lee and Shaffer [10] firstly introduced the slip-line field method to solve the orthogonal cutting problems. With this model, a unique solution is obtained by assuming the material in the uniform stress field to be in a state of incipient plasticity. In this model, the two systems of slip lines ( $\zeta$ and $\eta$ lines) are straight and inclined at $\pi / 2 \mathrm{rad}$ with each other. Lee and Shaffer's model reveals the basic mechanics of chip formation, although some simplifications are made in this model. When the chip upcurling and chip backflow effects were concerned for machining with a restricted contact tool, Fang et al. [11-13] developed a universal slip-line model, which produces a non-unique solution for the machining process. Oxley [3, 14] developed the "parallel-sided shear zone" model which is much closer to the real cutting process, since the effects of the strain hardening and thermal softening are involved. In this model, the slip line along the direction of primary shear plane is set to be $\zeta$ line and in the secondary shear zone the line along the tool face is set to be $\eta$ line. Though these two lines may not be orthogonal, the hydrostatic stresses along the slip lines are obtained based on the assumption of the two slip-line systems being orthogonal. More recently, Karpal and Özel $[15,16]$ proposed another new slip-line field model to investigate the thermomechanical cutting process and friction behavior of high speed machining.

It is noted that the available slip-line field models focus on pressure-independent materials where the two systems of the slip lines are assumed to be orthogonal. However, in recent years, the machining mechanics of some pressuresensitive materials, such as metallic glasses or glassy polymers, attract extensive attentions worldwide for their wide functional and structural applications. For example, Bakkal et al. [17, 18] investigated the chip light emission and morphology, surface roughness, and tool wear in cutting of Zr-based bulk metallic glass; Jiang and Dai [19] analyzed the stability of the process of machining metallic glass. It should be noticed that the plastic yield of the pressure-sensitive materials dependents not only on deviatoric stress but also on hydrostatic stress or pressure. When these pressure-sensitive materials are compressed, the yield stress along the shear planes is not constant but pressure dependent, and the two slip-line systems are nonorthogonal [20]. For example, the angle between the two slip-line systems could be lower than $79^{\circ}$ for some metallic glasses [21] and be smaller than $83^{\circ}$ for some polymers [20], which means that the pressure could make a significant influence on their yielding behavior. Therefore, the deformation of pressure-dependent material is much more complicated than that of pressure-independent material. In the excellent work of Hill [22], the slip-line theory was developed to study the plastic deformation of pressuredependent material, where the relations of stresses along the slip lines are established. However, when it comes to the problem of machining, new model is necessary to be developed for describing the process of cutting pressuredependent materials.

In this work, focusing on revealing the basic mechanics of machining pressure-dependent material, a simplified slip-line field model for orthogonal cutting of pressuresensitive materials is proposed. And the influence of the material pressure sensitivity on the cutting process is examined. Furthermore, the finite element model (FEM) simulation is performed for investigating the effects of the material and cutting parameters on the cutting process. A comparison of the predicted shear angle and cutting force with the FEM simulation results is carried out to validate the theoretical model.

\section{New slip-line field model}

\subsection{The yield criterion}

The material of the workpiece is assumed to be pressuresensitive material and its yield follows Mohr-Coulomb law,

$\tau=C_{0}+\sigma \cdot \tan \theta_{0}$

where, $\tau$ is shear stress, $\sigma$ normal stress, $C_{0}$ material cohesion strength, and $\theta_{0}$ material internal friction angle which determines the pressure or normal stress sensitivity. When this material yields, the angle between the two slipline systems can be defined as:

$\xi=\pi / 2-\theta_{0}$

During machining, the materials are always compressed, hence we set the pressure to be positive and further take the cutting process as a plane strain process. So, we can set the maximum shear stress $\tau$ whose magnitude is equal to the 
Mohr circle's radius $r$ (see Fig. 1) and the hydrostatic pressure $p$ as:

$\tau=\frac{1}{2}\left(\sigma_{1}-\sigma_{3}\right)$

$p=\frac{1}{2}\left(\sigma_{1}+\sigma_{3}\right)$

When materials yielding, $\tau$ and $p$ satisfies the following equation:

$\tau=C_{0} \cos \theta_{0}+p \sin \theta_{0}$

\subsection{The new slip-line field model}

In order to construct the slip-line field model for machining pressure-dependent materials, the orthogonal cutting condition is assumed and some other premises are made as follows:

1. As did in the previously developed models, the elastic strain of workpiece material is very small compared to the plastic strain during cutting, which can thus be neglected in cutting process analysis

2. The material ahead of the tool is taken as rigid and at rest.

3. When the material yields, the plastic deformation is assumed to be concentrated along a single shear plane which itself is a slip line in the 2-D analysis, and the another slip-line system should be inclined at $\xi$ radians with it.

4. All the slip lines are straight since the chip upcurling effect is neglected in the present analysis.

5. The chip is free as it leaves the plastic region.

Thus, the slip-field model for 2-D machining pressuresensitive materials which meets the operations as mentioned above can be established as shown in Fig. 2. It is found from Fig. 2 that both sets of slip lines are straight and the two systems are parallel to $A B$ and $C D$, respectively.

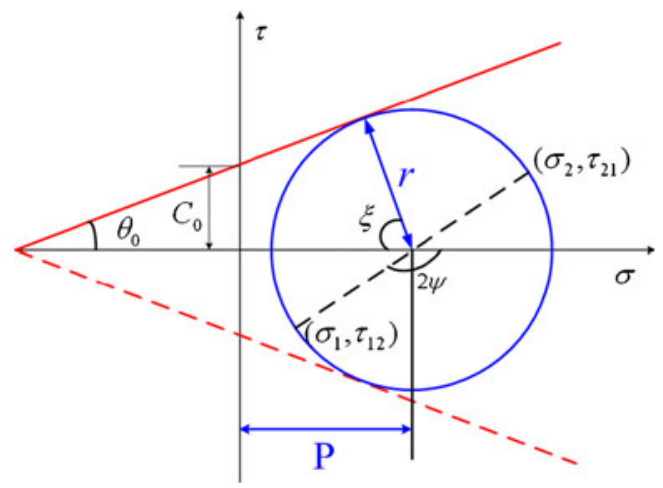

Fig. 1 Yield criterion of Mohr-Coulomb

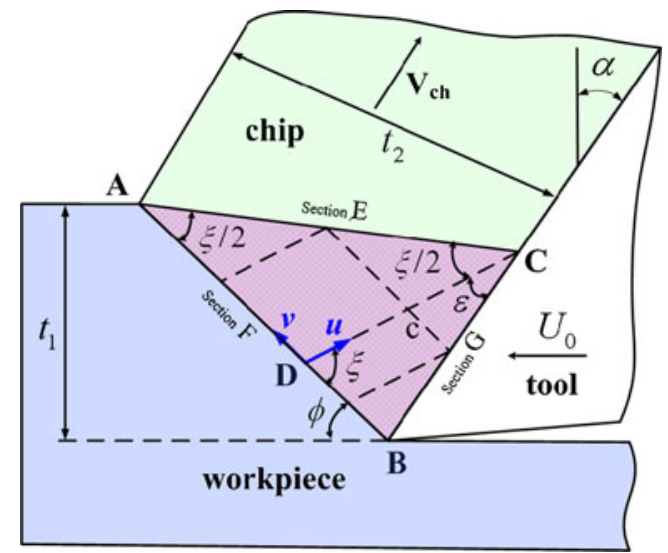

Fig. 2 The slip-line field model for orthogonal cutting with pressuresensitive material using flat-faced tool

The plastic flow occurs entirely across the shear line $A B$. The triangle field $A B C$ is a fictitious plastic field which is the limit of a rigid region. This field is used to examine the transmission of the machining force between the tool face and the shearing surface $A B$.

According to the present model, the chip above $A C$ is in stress-free state, so no stress is transmitted across $A C$. The zero shear stress across $A C$ and the yield limit of the material determines that all the slip lines are inclined at $\xi / 2$ with $A C$ rather than $\pi / 4$ in the previous slip-line field models (e.g., [10]). The other factors determining the configuration of the field is the tool's rake angle $\alpha$, and $\angle \mathrm{DCB}=\varepsilon$, which will be shown to be associated with both the friction conditions at the tool face and the material internal frictional angle. Since the slip lines are straight, the stress distribution is uniform in $A B C$, thus can be studied by means of Mohr's circle diagram which is shown in Fig. 3.

Since $A C$ is a force-free boundary, the Mohr circle should pass through the origin. In addition, according to the yield criterion, the Mohr circle should be tangent to the line $M N$, on which the stresses condition satisfies Eq. 1. The

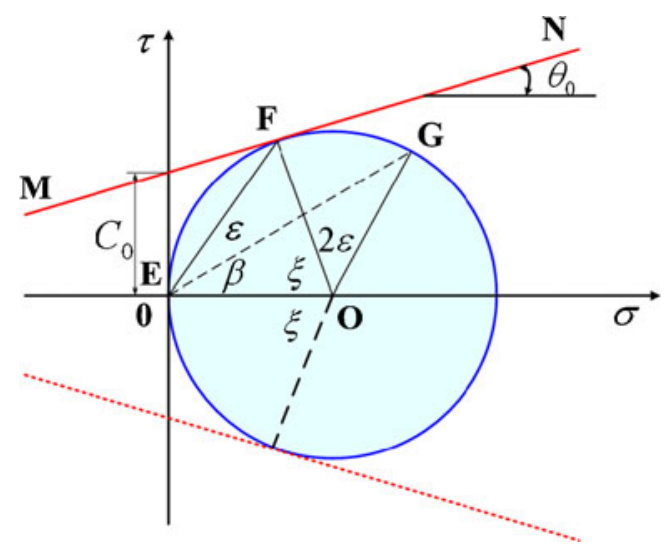

Fig. 3 The corresponding Mohr's circle diagram (compression is set to be positive) 
points marked in Fig. 3 represent the stress condition on the corresponding sections in Fig. 2. The traction at the tool face $B C$ is given by the point $G$, the ordinate of which denotes the shear stress, and the abscissa the normal stress. The ratio of these two stresses characterizes the coefficient of friction at the tool face, $\mu_{t}=\tan \beta$. So that $\beta$, the angle of friction between the chip and tool face, is given by $<$ OEG. And, according to the construction of the Mohr's circle diagram, $\angle \mathrm{FOG}=2 \varepsilon$; where, $\varepsilon$ is equal to the angle $\angle \mathrm{DCB}$ in Fig. 2. Since the angle subtended by a chord at the circumference is one half the angle subtended at the center, $<\mathrm{FEG}=\varepsilon$. Also $\angle \mathrm{EOF}=\xi$, so that

$\varepsilon=\frac{\pi}{4}-\beta+\frac{\theta_{0}}{2}$

Let us now consider the magnitude of the shear angle which has received considerable attention in the study of machining [1-10]. In the slip-line model, $C D$ is inclined at the angle $\varepsilon+\alpha$ with the vertical, and this is equal to $\phi+\pi / 2-\xi$ since $A B$ is inclined at the angle $\xi$ with $C D$. Thus, using Eq. 5, we have the analytical expression for the shear angle:

$\phi=\frac{\pi}{4}-\beta+\alpha-\frac{\theta_{0}}{2}$

This equation shows that the shear angle $\phi$ increases with increase in the rake angle $\alpha$, and decreases with decrease in friction angle $\beta$. This is the same as the Lee and Shaffer's solution for pressure-independent materials, where the shear angle is given by $\phi=\pi / 4-\beta+\alpha$. However, the shear angle calculated from the new model decreases linearly with the internal friction angle increasing, which is $\theta_{0} / 2 \mathrm{rad}$ smaller than that of Lee and Shaffer's solution for the identical $\alpha$ and $\beta$.

By making use of Eqs. 2, 5, and 6, the chip thickness $t_{2}$, shown in Fig. 2, can be obtained as

$t_{2}=t_{1} \frac{\cos \left(\frac{\pi}{4}-\beta-\frac{\theta_{0}}{2}\right)}{\sin \left(\frac{\pi}{4}-\beta+\alpha-\frac{\theta_{0}}{2}\right)}$

where, $t_{1}$ is the cutting depth. Figure 4 shows the variation of the dimensionless chip thickness ratio $t_{2} / t_{1}$ with the material internal friction angle. It can be found from this figure that the chip thickness $t_{2}$ increases with $\theta_{0}$ increasing for a fixed cutting depth $t_{1}$. This means that increasing the internal friction angle intensifies the shear deformation of the chip.

Now, let us consider the velocity distribution. The velocity components $u$ and $v$ along the slip lines are shown in Fig. 2. The tool is moving to the left with velocity $U_{0}$ and the workpiece below $A B$ is at rest. Continuity of the material across $A B$ requires the normal component of the velocity of the particles on either side of this line be equal. Since the velocity of the material below $A B$ is zero, the velocity components $u$ of material on $A B$ is also zero. For straight slip
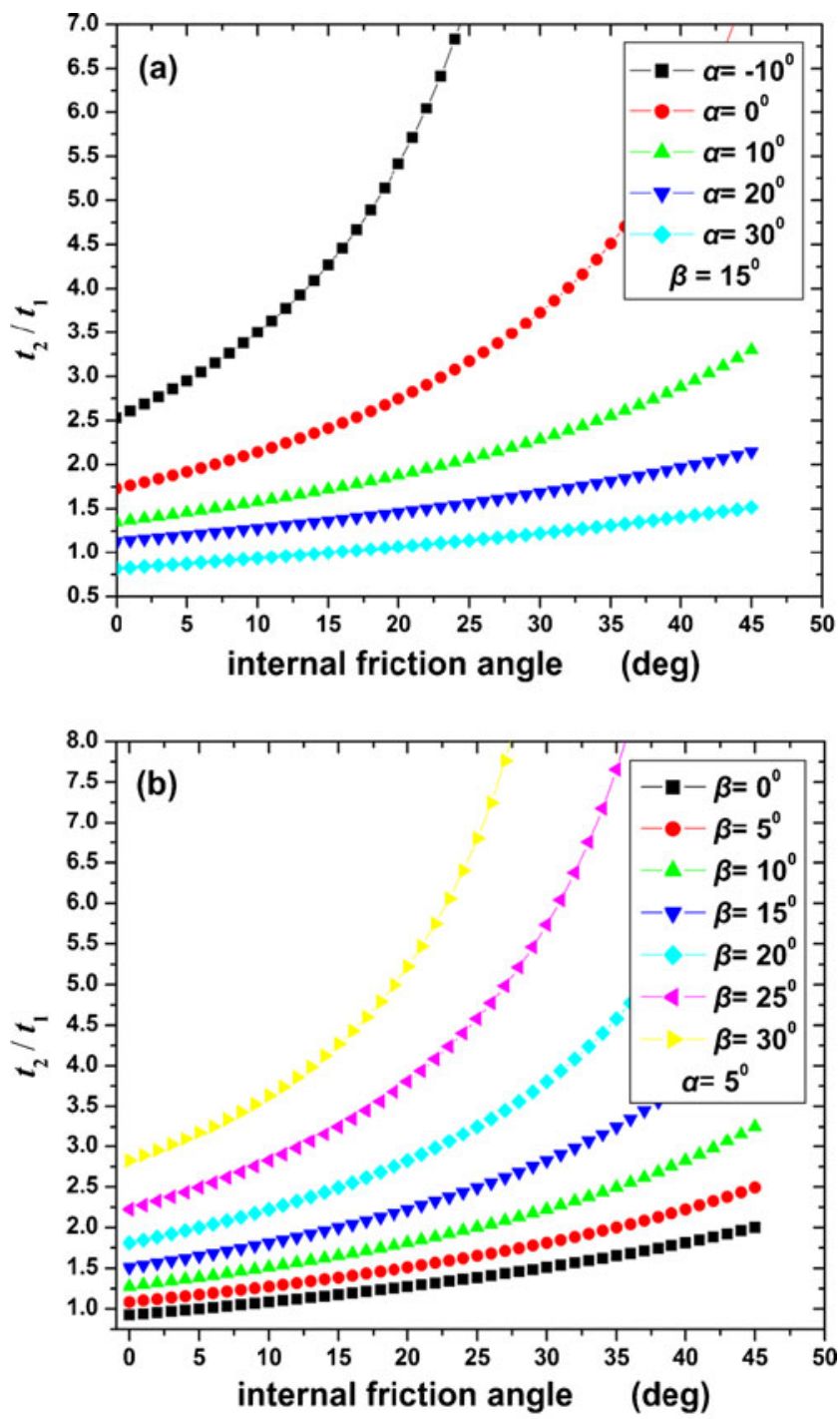

Fig. $4 t_{2} / t_{1}$ plotted with $\theta_{0}$ a for different rake angle where $\beta$ is $15^{\circ}, \mathbf{b}$ for different friction angle where $\alpha$ is $5^{\circ}$

lines, according to the Geiringser's equations (velocity equilibrium equations for slip lines), $u$ throughout the field $A B C$ is zero. Moreover, since the tool and chip are in contact, the component of the velocity of each, normal to the contact surface $B C$, must be the same. As $u$ is zero, this requires

$v=U_{0} \frac{\cos \alpha}{\cos \left(\frac{\pi}{4}-\beta-\frac{\theta_{0}}{2}\right)}$

Across $A B$ there is a discontinuity of magnitude $v$ in the tangential component of velocity. It is this discontinuity of velocity, corresponding to a line of infinite strain rate, which produces the plastic deformation in the chip. Moreover, because $u$ and $v$ are both constant in $A B C$, it moves as a rigid body, and no additional plastic strain in it. The continuity of the velocity across $A C$ requires the motion of the chip be the same as that of $A B C$, so that the chip slides up the tool face $B C$ straightly without curling. 
On the basis of the velocity solution, the deformation of an initially square network embedded in the workpiece material can be obtained. Figure 5a shows a particular case with $\beta=30^{\circ}, \alpha=30^{\circ}$, and $\theta_{0}=0^{\circ}$. It can be seen that simple shear occurs across the shear line and because of the constant velocity $u$ and $v$ in $A B C$, the chip is sheared uniformly above this line. When the material internal friction angle increases to $30^{\circ}$, the deformation of the network is shown in Fig. 5b, where the chip shears more intensively, and the deformation of the grids become more serious.

\section{The influence of the material pressure sensitivity on the cutting process}

Let us firstly consider the stresses along the shear plane and the tool face. As shown in Fig. 3, the magnitude of the

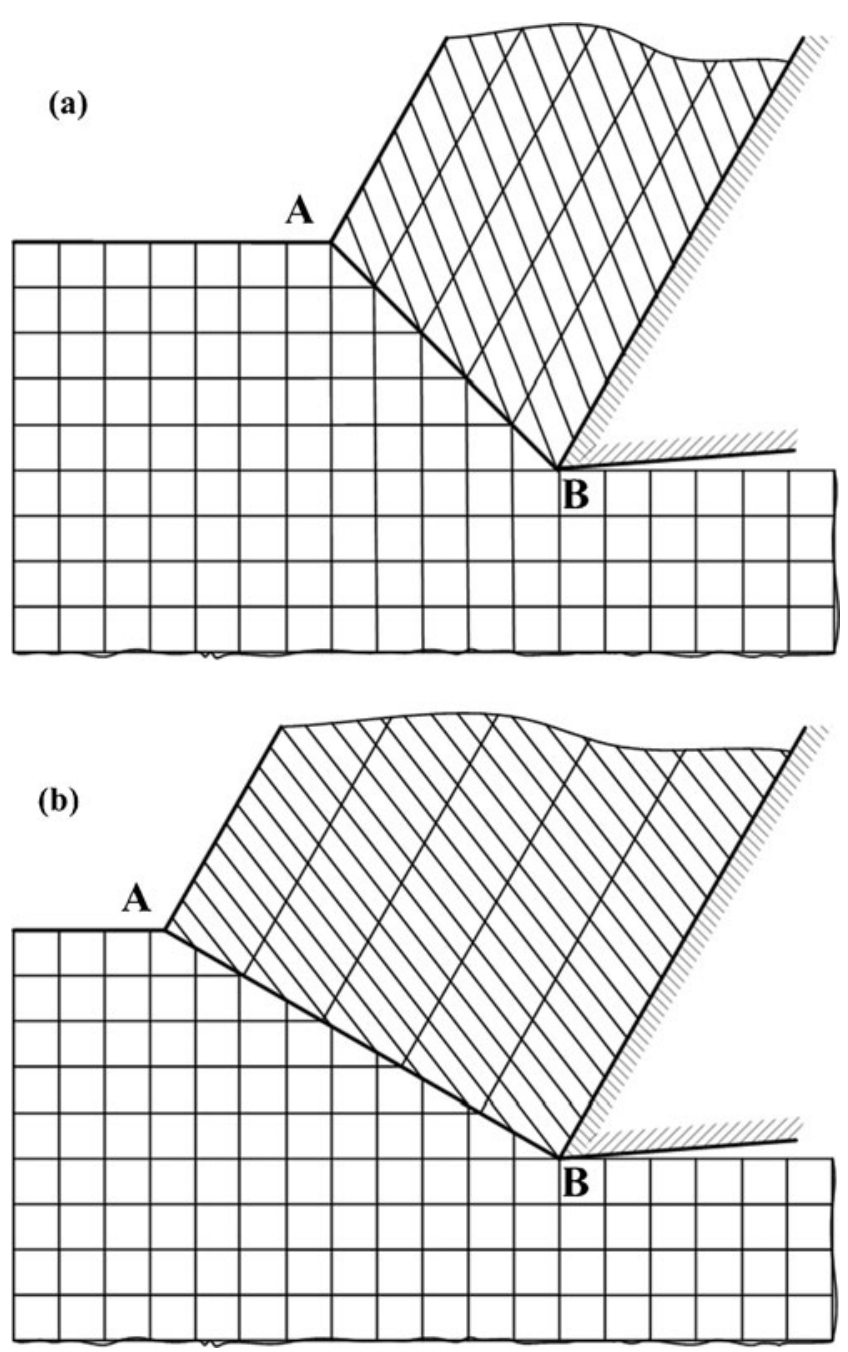

Fig. 5 Deformation of an initially square grid with $\beta=30^{\circ}, \alpha=30^{\circ}$, and $\mathbf{a} \theta_{0}=0^{\circ}, \mathbf{b} \theta_{0}=30^{\circ}$ hydrostatic pressure $p$ is equal to the Mohr's circle's radius $r$, using Eq. 4, we can get the hydrostatic pressure $p$ and the maximum shear stress $\tau$ as:

$p=\tau=C_{0} \frac{1+\sin \theta_{0}}{\cos \theta_{0}}$

Thus, the shear stress $\tau_{n}$ and normal stress $\sigma_{n}$ along the shear line $A B$ can be determined.

$\tau_{n}=C_{0}\left(1+\sin \theta_{0}\right)$

$\sigma_{n}=C_{0} \cos \theta_{0}$

Also, the normal stress $\sigma_{\text {int }}$ and shear stress $\tau_{\text {int }}$ along the tool rake can be obtained from the point $\boldsymbol{G}$ in Fig. 3 as:

$\sigma_{\text {int }}=C_{0} \frac{\left(1+\sin \theta_{0}\right)(1+\cos (2 \beta))}{\cos \theta_{0}}$

$\tau_{\text {int }}=C_{0} \frac{\left(1+\sin \theta_{0}\right) \sin (2 \beta)}{\cos \theta_{0}}$

The total force on the tool can be obtained based on the present theory that $A B C$ is in equilibrium and the tool force is equal to the force transmitted across $A B$. The horizontal component of the cutting force on the tool is the most significant force variable, for it determines the power expended in machining. For the depth of cut is $t_{1}$, and the shear and normal stresses on $A B$ can be determined from Eqs. 10.a, 10.b; the cutting force $F_{c}$ can be written as:

$F_{c}=C_{0} t_{1}\left[\left(1+\sin \theta_{0}\right) \cot \left(\frac{\pi}{4}-\beta+\alpha-\frac{\theta_{0}}{2}\right)+\cos \theta_{0}\right]$

The thrust force $F_{t}$, normal to the cutting direction, can also be obtained by the corresponding component of the traction on $A B$,

$F_{\mathrm{t}}=C_{0} t_{1}\left[\cos \left(\theta_{0}\right) \cot \left(\frac{\pi}{4}-\beta+\alpha-\frac{\theta_{0}}{2}\right)-\left(1+\sin \theta_{0}\right)\right]$

Next, let's discuss about the influence of the material internal friction angle $\theta_{0}$ on the cutting force. In Fig. 6, $\left(F_{c}\right) /\left(C_{0} t_{1}\right)$ are plotted as a function of the internal friction angle $\theta_{0}$ for various rake angle $\alpha$ and friction angle $\beta$, respectively. It can be seen from Fig. 6 that, for a fixed internal friction angle, the cutting force $F_{c}$ decrease with the rake angle $\alpha$ increasing or the friction angle $\beta$ decreasing. These results agree with that of Lee and Shaffer [10]. Furthermore, it seems clear from Fig. 6 that, for the same cutting condition, the machining force increases with increased in the material internal friction angle for the identical cohesion. This means that machining the material 

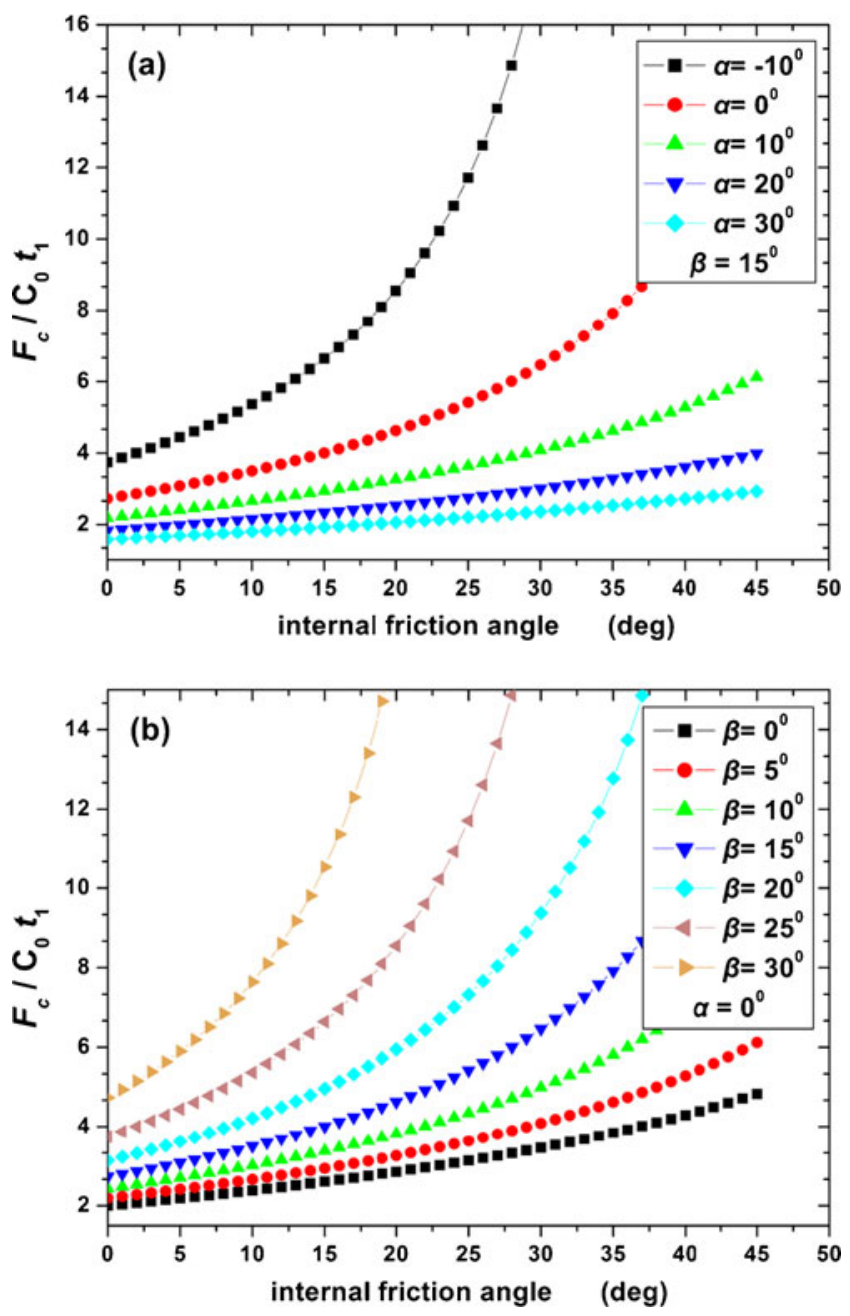

Fig. $6 F_{c} / C_{0} t_{1}$ plotted with $\theta_{0}$ a for different rake angle where $\beta$ is $15^{\circ}, \mathbf{b}$ for different friction angle where $\alpha$ is $0^{\circ}$

with higher internal friction angle could be much harder. In addition, the influence of the internal friction angle on the cutting force is significant when $\alpha$ is small and $\beta$ is high. So, if the rake angle is negative and the tool-chip friction is severe, considering the material pressure-sensitive effect is of special significant. For some metallic glasses, the internal friction angle could be larger than $10^{\circ}$ under compression [21]. Thus, when these metallic glasses are machined with a rake angle of $-10^{\circ}$, the material pressure sensitivity could lead to an increase of $50 \%$ in cutting force.

Now, we discuss how the internal friction angle affects the cutting force and shear angle. For a given internal friction angle, once the stable cutting process is established, the shear angle and cutting force are fixed. If there is a sudden increase of the internal friction angle, the friction between the chip and workpiece will increase immediately. Keep in mind that the cutting force does not change yet at the very beginning of increasing the internal friction angle. So that the shear stress along the shear plane is not large enough to overcome the addition friction. Therefore, in order to deform the workpiece into chip, the cutting force increases to make sure that the shear stress being large enough to yield the workpiece material. This will lead to an increase of cutting energy. However, according to Piispanen [4] and Merchant [6], the shear angle will change itself to reduce the additional friction and hence to minimize the increase of cutting energy. The friction between the workpiece and chip can be written as

$f=\frac{\sigma_{n} t_{1}}{\sin \phi \tan \theta_{0}}=\left(F_{c} \sin \phi-F_{t} \cos \phi\right) \tan \theta_{0}$

Usually, $\left|F_{c}\right| \gg\left|F_{t}\right|$, so the friction $f$ is approximately equal to $F_{c} \sin \phi \tan \theta_{0}$. As discussed above, increasing the internal friction angle $\theta_{0}$ increases the cutting force $F_{c}$. Thus, in order to reduce the additional friction, it needs a decrease of the shear angle. And according to Eq. 6, the decrease of shear angle should be equal to a half of the internal friction angle increment.

\section{Comparison of the theoretical results with the FEM simulation}

To investigate the effect of the material properties and cutting parameters on the cutting process, the finite element simulation is performed as well.

Arbitrary Lagrangian Eulerian (ALE) technology is widely used in the modeling of orthogonal metal cutting process. Movahhedy et al. [23] and Adetoro and Wen [24] have shown that due to the characteristics of the cutting process, the ALE formulation offers the most efficient modeling approach. Moreover, by using the ALE technology, artificial criteria (mechanical or geometrical) to generate the chip formation is not needed, as material can be deformed without mesh distortion, and the chip can separate from the workpiece by mesh remeshing. So, in this work, the FEM using ALE formulation proposed in ABAQUS/Explicit is employed to predict the shear angle and cutting forces during cutting process. Furthermore, followed by the excellent work of Mamalis et al. [25] and Paulo et al. [26], which applied 2-D FEM to chip formation in orthogonal cutting, the modeling is performed two dimensionally under plane strain conditions. Four-node plane strain quadrilateral elements with reduced integration and hourglass control are used in the workpiece.

Table 1 Cutting parameters

\begin{tabular}{lllll}
\hline $\begin{array}{l}\text { Cutting } \\
\text { speed } U_{0} \\
(\mathrm{~m} / \mathrm{s})\end{array}$ & $\begin{array}{l}\text { Workpiece } \\
\text { thickness } \\
(\mathrm{mm})\end{array}$ & $\begin{array}{l}\text { Cutting } \\
\text { depth } t_{1} \\
(\mathrm{~mm})\end{array}$ & $\begin{array}{l}\text { Tool rake } \\
\text { angle } \alpha \\
(\mathrm{deg})\end{array}$ & $\begin{array}{l}\text { Tool-chip } \\
\text { friction angle } \\
\beta(\mathrm{deg})\end{array}$ \\
\hline 4 & 1 & 0.2 & -10 & 10 \\
\hline
\end{tabular}


Fig. 7 The equivalent plastic strain for a $\theta_{0}=0^{\circ}, \mathbf{b} \theta_{0}=5^{\circ}, \mathbf{c}$ $\theta_{0}=10^{\circ}, \mathbf{d} \theta_{0}=15^{\circ}$
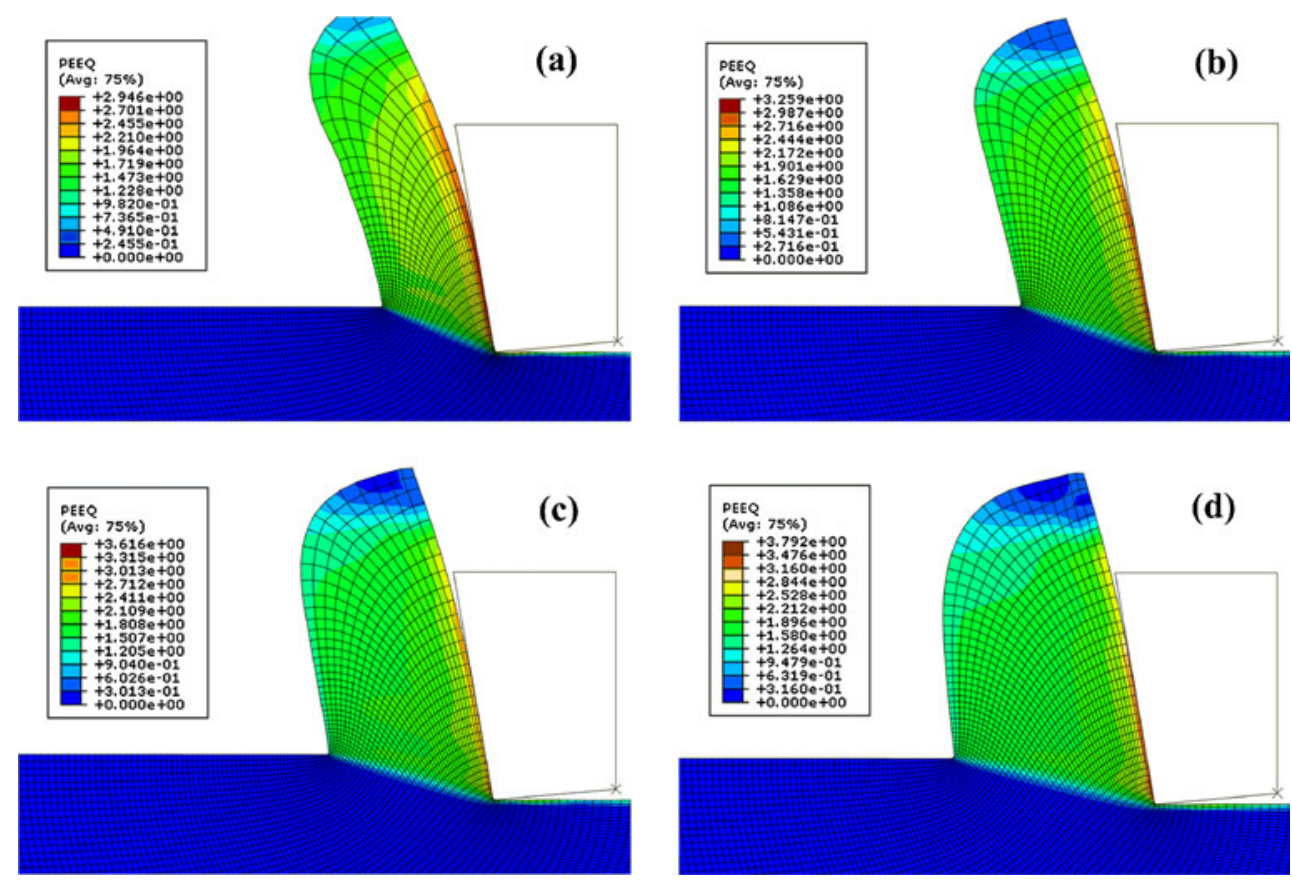

(d)
The tool is modeled as rigid bodies with zero radii, which has a rake angle $\alpha=-10^{\circ}$ for ideally sharp tool and a clearance angle $\gamma=5^{\circ}$. The contact between tool and chip is modeled as sliding friction with a friction angle $\beta=10^{\circ}$. As to make the cutting conditions as close as in our theoretical model, the flow stress of the workpiece was set to be independent with temperature, strain, and strain rate but just modeled with an elasto-plastic constitutive model which followed the yield criterion of Mohr-Coulomb. The cohesion was set to be $400 \mathrm{MPa}$ as a constant and the internal friction angle was set between $0^{\circ}$ and $20^{\circ}$. So far, the other cutting parameters are held constant in this simulation, only the work material internal friction angle $\theta_{0}$ was varied from $0^{\circ}$ to $20^{\circ}$. The cutting parameters are shown in Table 1.

The equivalent plastic strain and chip geometry for internal friction angle equal to $0^{\circ}, 5^{\circ}, 10^{\circ}$, and $15^{\circ}$ after the cutting process stabilized are shown in Fig. 7. From the figure, we can easily see that increasing the material internal friction angle broadens the chip, lowers the shear angle, and finally leads to a higher cutting force as showin in Table 2.

Figure 8 a shows the variation of the shear angle with respect to the internal friction angle for the numerical and theoretical result. A comparison of the simulated result with the theoretical result shows a remarkable similarity. However, for each internal friction angle, the shear angle of the

Table 2 Simulation results

\begin{tabular}{lccccc}
\hline Internal friction angle & $\theta_{0}=0^{\circ}$ & $\theta_{0}=5^{\circ}$ & $\theta_{0}=10^{\circ}$ & $\theta_{0}=15^{\circ}$ & $\theta_{0}=20^{\circ}$ \\
\hline Shear angle (deg) & 22.6 & 19.8 & 17.2 & 14.4 & 11.7 \\
Cutting force (N) & 274 & 322 & 374 & 456 & 578 \\
\hline
\end{tabular}

simulated value is about $2-3^{\circ}$ smaller than the theoretical result. This may be caused by some differences between the theoretical model and FEM model, such as the elastic deformation, the chip upcurling effect, and the shear deformation along the tool face, which is not considered in the theoretical analysis but could happen in the numerical simulation.

In Fig. 8b, the simulation result of specific cutting pressure (cutting force divided by workpiece thickness and cutting depth) against the internal friction angle is com-
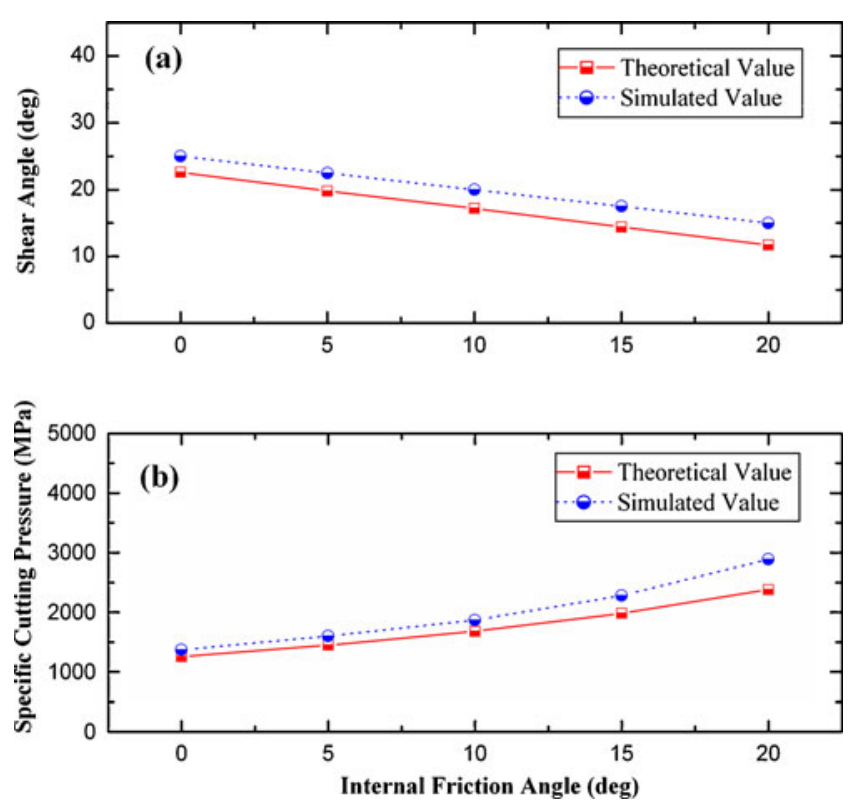

Fig. 8 a The shear angle and $\mathbf{b}$ the specific cutting force as a function of the internal friction angle for theoretical and simulated results 
pared with the theoretical result calculated from Eq. 12. The simulated result is plotted as a solid line and the theoretical result dashed. It shows a reasonably good match of these two lines. However, since the simulated shear angle is smaller than the theoretical value, the numerical result of the specific cutting force is little bigger than the theoretical result, as the solid line is little higher than the dashed one.

The comparison between the theoretical and numerical results shows a good agreement, which indicates that the material pressure sensitivity exerts a significant influence on the cutting process, namely, the material become more hard to machine with the material internal friction angle increasing. The comparison of the theoretical and numerical results also shows that the new slip-line filed method can characterize machining of the pressure-dependent materials satisfactorily.

\section{Conclusions}

In this work, a new straight slip-line filed model for orthogonal machining pressure-sensitive materials is proposed and the analytical expressions for shear angle, cutting force, and chip configuration are obtained.

Using this new model, the shear angle is obtained to be $\pi / 4-\beta+\alpha-\theta_{0} / 2$, which is $\theta_{0} / 2 \mathrm{rad}$ smaller than the Lee and Shaffer's solution and decreases linearly with the internal friction angle increasing. Furthermore, increasing the internal friction angle enlarges both the cutting force and chip depth, especially when the rake angle is small and the friction at the tool face is high. In a word, increasing the internal friction angle makes the machining much harder and more power consuming.

The finite element model using ALE formulation was employed to make comparison with the theoretical results. The numerical results of the FEM simulation show good agreement with the predicted shear angle and cutting force, which shows the validity of our new slip-line field model.

Acknowledgments This work has been supported by the National Basic Research Program of China (grant no: 2009CB724401), the Nature Science Foundation of China (grants nos. 10725211, 11021262 and 11002144), and the National Natural Science Foundation of China-NSAF, grant no: 10976100.

\section{References}

1. Atkins AG (2009) The science and engineering of cutting: the mechanics and processes of separating, scratching and puncturing biomaterials, metals and non-metals. Butterworth-Heinemann, Boston

2. Shaw MC (2005) Metal cutting principles, 2nd edn. Oxford University Press, Oxford

3. Oxley PLB (1989) Mechanics of machining: an analytical approach to assessing machinability. Wiley, New York
4. Piispanen V (1948) Theory of formation of metal chips. J Appl Phys 19:876-881

5. Merchant ME (1945) Mechanics of the metal cutting process. I: orthogonal cutting and the type 2 chip. J Appl Phys 16:267-275

6. Merchant ME (1945) Mechanics of the metal cutting process II: plasticity conditions in orthogonal cutting. J Appl Phys $16: 318-324$

7. Atkins AG (2003) Modelling metal cutting using modern ductile fracture mechanics quantitative explanations for some long standing problems. Int J Mech Sci 45:373-396

8. Atkins AG (2005) Toughness and cutting: a new way of simultaneously determining ductile fracture toughness and strength. Eng Fract Mech 72:849-886

9. Subbiah S, Melkote SN (2007) Evaluation of Atkins' model of ductile machining including the material separation component. J Mater Process Technol 182:398-404

10. Lee EH, Shaffer BW (1951) The theory of plasticity applied to a problem of machining. Trans ASME: J Appl Mech 73:405-413

11. Fang N, Jawahir IS, Oxley PLB (2001) A universal slip-line model with non-unique solutions for machining with curled chip formation and a restricted contact tool. Int J Mech Sci 43:557-580

12. Fang N, Jawahir IS (2001) A new methodology for determining the stress state of the plastic region in machining with restricted contact tools. Int J Mech Sci 43:1747-1770

13. Fang N, Jawahir IS (2002) Analytical predictions and experimental validation of cutting force ratio, chip thickness, and chip backflow angle in restricted contact machining using the universal slipline model. Int J Mach Tools Manuf 42:681-694

14. Oxley PLB, Hstings WF (1977) Predicting the strain-rate in the zone of intense shear in which the chip is formed in machining from the dynamic flow stress properties of the work material and the cutting conditions. Proc R Soc London A 356:395-410

15. Karpal Y, Özel T (2008) Analytical and thermal modeling of highspeed machining with chamfered tools. Trans ASME: J Manuf Sci Eng 130:011001

16. Karpal Y, Özel T (2008) Mechanics of high speed cutting with curvilinear edge tools. Int J Mach Tools Manuf 48:195-208

17. Bakkal M, Shih AJ, Scattergood RO (2004) Chip formation, cutting forces, and tool wear in turning of $\mathrm{Zr}$-based bulk metallic glass. Int J Mach Tools Manuf 44:915-925

18. Bakkal M, Shih AJ, McSpadden SB, Scattergood RO (2005) Thrust force, torque, and tool wear in drilling the bulk metallic glass. Int J Mach Tools Manuf 45:863-872

19. Jiang MQ, Dai LH (2009) Formation mechanism of lamellar chips during machining of bulk metallic glass. Acta Mater 57:2730-2738

20. Jeong HY, Li XW, Yee AF, Pan J (1994) Slip lines in front of a round notch tip in a pressure-sensitive material. Mech Mater 19:29-38

21. Yu GS, Lin JG, Mo M, Wang XF, Wang FH, Wen CE (2007) Effect of relaxation on pressure sensitivity index in a $\mathrm{Zr}$-based metallic glass. Mater Sci Eng A 460:58-62

22. Hill R (1950) The mathematical theory of plasticity. Clarendon, Carolina, pp 294-300

23. Movahhedy M, Gadala MS, Altintas Y (2000) Simulation of the orthogonal metal cutting process using an arbitrary LagrangianEulerian finite-element method. J Mater Process Technol 103:267-275

24. Adetoro OB, Wen PH (2010) Prediction of mechanistic cutting force coefficients using ALE formulation. Int $\mathrm{J}$ Adv Manuf Technol 46:79-90

25. Mamalis AG, Kundr J, Markopoulos A, Manolakos DE (2008) On the finite element modeling of high speed hard turning. Int J Adv Manuf Technol 38:441-446

26. Paulo DJ, Maranhao C, Jackson MJ, Cabral G, Gracio J (2008) FEM analysis in high speed machining of aluminium alloy (A17075-0) using polycrystalline diamond (PCD) and cemented carbide (K10) cutting tools. Int J Adv Manuf Technol 39:1093-1100 